\title{
Signatures of processing complexity during global cognitive states in ventromedial prefrontal cortex
}

\author{
Priyanka S. Mehta ${ }^{1}$, Seng Bum Michael Yoo ${ }^{1,2,3}$, and Benjamin Y. Hayden ${ }^{1}$
}

1. Department of Neuroscience, Center for Magnetic Resonance Research, Department of Biomedical Engineering, and Center for Neuroengineering, University of Minnesota, Minneapolis MN 55455

2. Center for Neuroscience Imaging Research, Institute for Basic Science, Suwon, Republic of Korea, 16419.

3. Current address: Department of Brain and Cognitive Sciences, Massachusetts Institution of Technology, Cambridge, Massachusetts, MA, 02139

*Corresponding author:

Seng Bum Michael Yoo

Center for Neuroscience Imaging Research, Institute for Basic Science and Department, Suwon, Republic of Korea, 16419

Email address: sbyoo.ur.bcs@gmail.com

\section{Keywords}

Foraging, inter-trial interval, search, dimensionality, decodability

\section{Acknowledgements}

This work is supported by a R01 from NIH (DA037229) to BYH. We thank Meghan Castagno, Marc Mancarella, and Giuliana LoConte for assistance with data collection, and the rest of the Hayden lab for valuable discussions.

\section{Conflict of interest}

The authors have no competing interests to declare. 


\section{ABSTRACT}

Behavioral neuroscience almost exclusively studies behavior during tasks and ignores the

38 unstructured inter-trial interval (ITI). However, it is unlikely that the ITI is simply an idling or

39 paused mode; instead, it is a likely time for globally focused cognition, in which attention is

40 disengaged from the task at hand and oriented more broadly. To gain insight into the

41 computational underpinnings of globally focused cognition, we recorded from neurons in a core

42 decision-making region, area 14 of ventromedial prefrontal cortex (vmPFC), as macaques

43 performed a foraging search task with long inter-trial intervals (ITIs). We find that during the

44 ITI, ensemble firing is associated with increased discriminability of a key mnemonic variable,

45 recent reward rate, which in turn predicts upcoming search strategy. ITI activity is also

46 associated with increased ensemble dimensionality and faster subspace reorganization, presumed

47 markers of processing complexity. These results demonstrate the flexible nature of mnemonic

48 processing and support the idea that the brain makes use of ostensible downtime to engage in

49 complex processing.

50 


\section{INTRODUCTION}

Our minds can readily switch between states that are stimulus-focused and oriented

54 narrowly on the task at hand (local states) and stimulus-independent states that are relatively

55 undirected and more attuned to the world around us (global states, Raichle, 2010; Raichle, 2015;

56 Mason et al., 2007; Smallwood et al., 2013). During local states, many of the task-irrelevant

57 things on our mind fall away so that we can selectively process the most important and urgent

58 information. When our thoughts move away from the task, our minds then fill with a variety of

59 stimulus-independent thoughts, including recollections of the recent past, as well as background

60 and off-task cognition (Smallwood et al., 2013; Baird et al., 2013; Konishi et al., 2015).

Globally oriented cognition is poorly understood. We know a lot more about what goes

62 on in the brain during brief periods of focused performance than during the often much longer

63 times between. However, far from being a mental equivalent of doing nothing, globally oriented

64 cognition appears to be rich and complex, and too extend both backwards and forwards in time

65 (Northoff et. al, 2010, Mazoyer et al., 2001; Zou et al., 2013; Cole et al., 2016 ). Indeed, even if

66 global cognition is not directly related to the specific details of the most recent trial, it is

67 associated with several functions, such as reactivating past sequences of action, guiding

68 navigation in future goal-direction movement, and maintaining cognitive control and working

69 memory (Olafsdottir et al., 2018; Raichle, 2010; Jilka et al., 2014; ). One theme that unites these

70 functions is that they have at least some mnemonic relevance. Specifically, they involve

71 reactivation of memories of the recent and distant past.

72 Global cognitive functions are particularly associated with a discrete network of brain

73 regions known as the default mode network (DMN). Hemodynamic activity in DMN regions is

74 enhanced during delays between trials and in the absence of specific task instructions (Raichle et 
75 al., 2015; Behrens et al., 2018; Hayden et al., 2009b) Activity in the DMN appears to reflect

76 cognitive processes that are antagonistic to task focus. For example, DMN activity predicts

77 sporadic lapses in attention (Weissman et al., 2006), failures to encode memories (Daselaar et al.,

78 2004), and failures to perceive near-threshold somatosensory stimuli (Boly et al., 2007). One

79 region of the DMN, the medial prefrontal cortex, is associated with an increased BOLD signal

80 when subjects are tasked with processing information from previous trials (Konishi et al. 2015)

81 including memory consolidation during stimulus-independent thought (Smallwood et al., 2013;

82 Baird et al., 2013). These observations support the idea that activation of mnemonic processes

83 naturally competes with effective task performance.

We wished to gain insight into the neural processes occurring in global cognition. We

85 examined ensemble firing rates in one DMN structure, the ventromedial prefrontal cortex

86 (vmPFC), as macaques performed a computerized foraging task. The task, like most laboratory

87 tasks, is naturally divided into a task period and an inter-trial interval (ITI). Our analyses

88 therefore centered on comparing neural activity during the task to activity during the ITI. We

89 used a computerized foraging task that dissociates behavioral modes in macaques. On each trial

90 (the presumed local state), the subject must search for several seconds between options that offer

91 obscured riskless but variable rewards. During the 4-second ITI subjects presumably enter into a

92 globally-oriented cognitive state. To gain insight into the role of vmPFC in global cognition, we

93 focused on a behavior known as adjustment. Adjustment is a form of rapid learning and may

94 reflect a strategic change motivated by an underlying belief that task statistics have changed

95 (Hayden et al., 2008; Hayden et al., 2009a; Hayden et al., 2011; Blanchard et al., 2015). We

96 hypothesized that the ITI may be a critical period for performing adjustment-related

97 computations. 
We find three striking differences between these two task phases. First, information about

99 the rate of recent reward intake (over the past 10 trials) is more readily distinguished in the ITI,

100 and that this information closely predicts subsequent adjustment. Second, neural ensemble

101 dimensionality is substantially greater during the ITI. Third, the change rate of the subspace

102 reorganization is significantly enhanced during the ITI. Together, these results highlight the

103 inherent richness of neuronal processing during the ITI, and during global cognition more

104 generally, and, in particular, suggest that this processing privileges mnemonically relevant

105 information. 


\section{RESULTS}

Macaques performed a foraging search task in which they inspected a series of hidden

offers and selected one of them to obtain a liquid reward (Figure 1A, Mehta et al., 2019). The

reward was deterministic but its value was randomized by offer and not predictable before

secretary problem and other stopping problems (Ferguson, 1989). Detailed behavior in the task is

113 (Mehta et al., 2019).

While our subjects performed the task, we recorded responses of 122 single neurons in

118 and the location of the attended offer (Mehta et al., 2019). While our previous study focused on

119 trial-related activity, here we focus primarily on comparing activity between the trial and the inter-trial interval (ITI). The key feature of our search task that enables this analysis is the

121 unusually long ITI (4 seconds), which allowed us to study responses temporally dissociated (>1

122 second) from both the past trial and the upcoming one, thus avoiding both sustained / hysteretic 123 and anticipatory effects.

We focused our analyses on two $500 \mathrm{~ms}$ epochs. The ITI epoch is centered on the

125 midpoint of the ITI - exactly two seconds after it begins and two seconds before it ends. This

126 means our window of analysis spans $250 \mathrm{~ms}$ before this point, and $250 \mathrm{~ms}$ after. Our trial epoch

127 is centered on the midpoint of the 1-second time period after the reveal of the first offer of each 
trial. This window of analysis spans from $250 \mathrm{~ms}$ after the offer reveal to $750 \mathrm{~ms}$ after the offer reveal.

A central Fixation

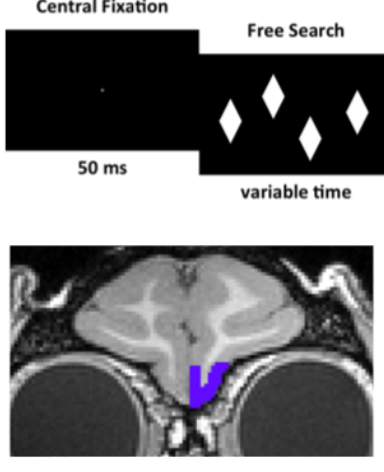
Diamond 1 Fixation

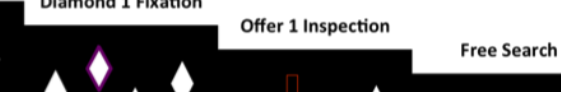

B

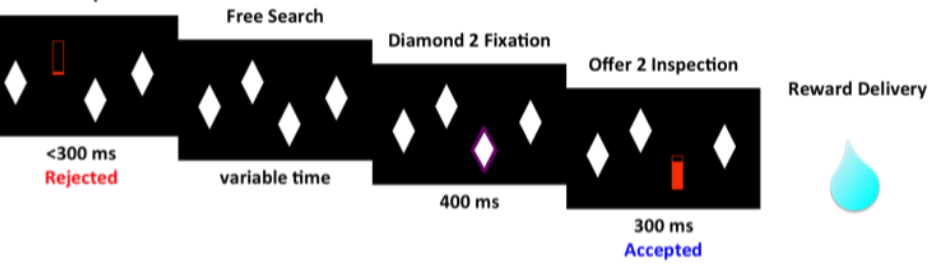

Figure 1. Our foraging task. (A) A typical trial. Subject searches through multiple offers and accepts one, ending the trial. (B) Area from which all recordings were obtained: macaque area 14, analogue of human ventromedial prefrontal cortex.

\section{Mnemonic information is more decodable during the ITI than during the task}

Our central hypothesis is that firing patterns during the inter-trial interval are neither

adjustment may reflect a strategic response to evidence that environmental richness has changed 
analysis excludes the most recent trial. Thus, all the events that drive the response come from 10-

15170 seconds before the choice is expressed.

Recent reward rate is robustly encoded by responses of vmPFC neurons. Indeed, during the $500 \mathrm{~ms}$ trial epoch, $22.13 \%$ of neurons in vmPFC encoded the average reward rate on the 154 past ten trials ( $18.57 \%$ and $26.92 \%$ in subjects $\mathrm{J}$ and $\mathrm{T}$, respectively). (As above, this and the 155 next analysis exclude the most recent trial to eliminate bias by possible behaviorally hysteretic 156 effects). A similar proportion of neurons (23.77\%) encode the same information during the 500 157 ms ITI epoch (J: 28.57\%; T:17.31\%). All four of these proportions are substantially greater than 158 chance (binomial test, $\mathrm{p}<0.0001$ in all cases).

We next hypothesized that, if ITI was associated with mnemonic function, then the recent 160 reward rate would be more informationally accessible during the ITI than during the task period.

161 To test this hypothesis, we first devised a measure of informational accessibility based closely on 162 a technique previously devised by Cohen and Maunsell (2010) and extended by Keemink and 163 Machens (2019). We used a dimensionality reduction approach; specifically, one that uses 164 Principal Component Analysis (PCA). We first projected our condition-averaged peri-stimulus 165 time histograms (which represent time binned vectors of firing) into a PCA space. That is, we 166 averaged all the peri-stimulus time histograms in an epoch across each condition (in this case, 167 high and low reward rate).

169 components that efficiently describe the population activity as it varies with reward rate. We

170 focused on the three principal components that describe the largest amount of neural activity for

171 each group of trials (high-reward-rate trials and low-reward-rate trials). This allowed us to

172 graphically represent the population activity: each principal component became an axis, with 
173

174

175

176

177

178

179

180

181

182

183

each point on the resulting three-dimensional plot representing a projection of the neural

population (Figure 2A). We compared the two sets of points - the neural population during lowreward-rate trials versus the neural population during high-reward-rate trials.

We were interested in the question of whether information is more separable (i.e., decodable) during the ITI than it is during the trial epoch (Cohen \& Maunsell, 2010). To determine the separability between high-reward-rate and low-reward-rate trials, we used a linear discriminant analysis (LDA) and calculated the distance ( $\left.d^{\prime}\right)$ between the cloud of high-rewardrate points and that of low-reward-rate points. A larger distance means a greater difference in the way the vmPFC population represents high vs. low reward rate. In other words, a downstream brain region with access to this information would have an easier time determining whether reward rate was high or low with a high $d^{\prime}$. We found that high and low reward rate trials were significantly more separable during the ITI than during the trial in both subjects (subject J: ITI epoch $d^{\prime}$ 6.97, trial epoch $d^{\prime}$ 0.63; $\mathrm{p}<0.001$, paired t-test; subject T: ITI epoch $d^{\prime} 5.17$, trial epoch $d^{\prime}$ 0.61, p $<0.001$, paired t-test; Figure 2A, Methods).

If the global reward rate is actively calculated during the ITI, then it should influence choice behavior during foraging. We previously reported that the behavioral threshold does change systematically with reward rate (Mehta et al., 2019). Here we demonstrate a significant difference between behavioral threshold over the same categories we found separation between during the ITI: high and low reward rate (subject J: low reward rate threshold $47.41 \%$, high reward rate threshold $51.50 \%, \mathrm{p}<0.01,100$-repetition bootstrap; subject $\mathrm{T}$ : low reward rate threshold 53.73\%, high reward rate threshold 58.89\%; $\mathrm{p}<0.01,100$-repetition bootstrap; Figure 2B, Methods). This illustrates a potential use for distinguishing high and low reward rate before the trial starts: adjusting behavioral strategy in accordance with reward rate. 


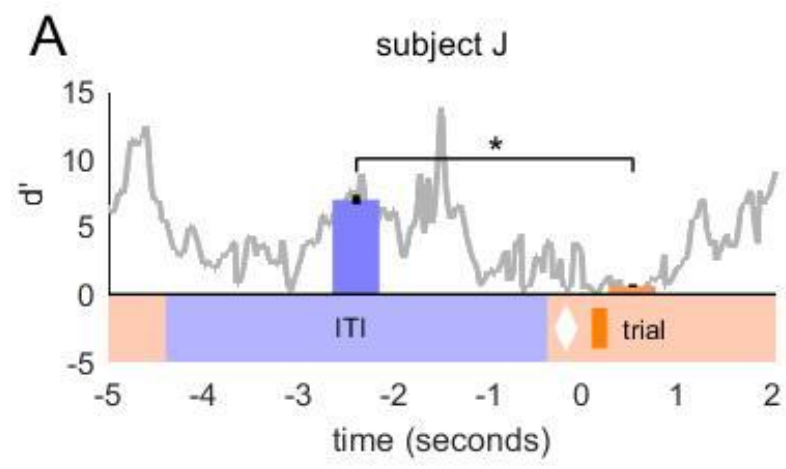

mid-ITI

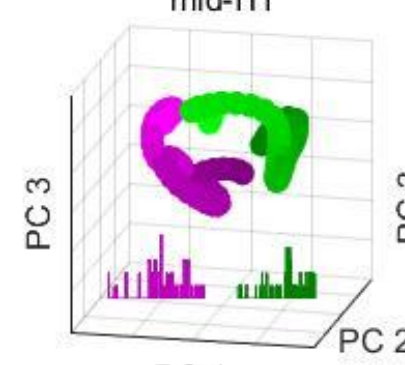

PC 1

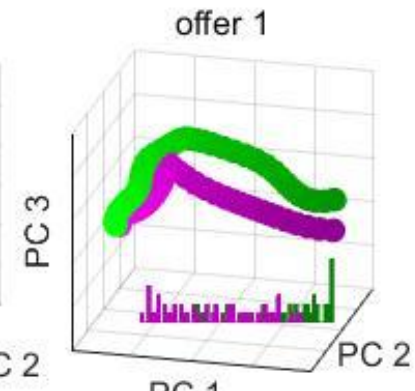

PC 1

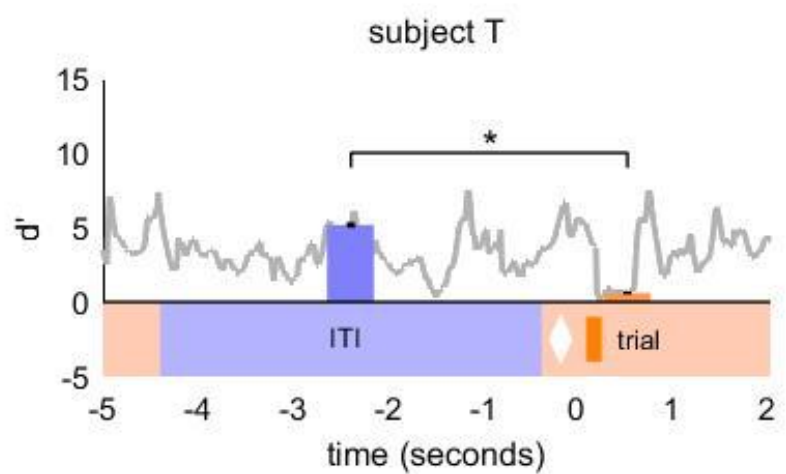

mid-ITI

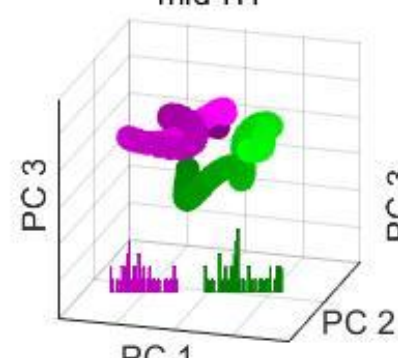

PC 1 offer 1

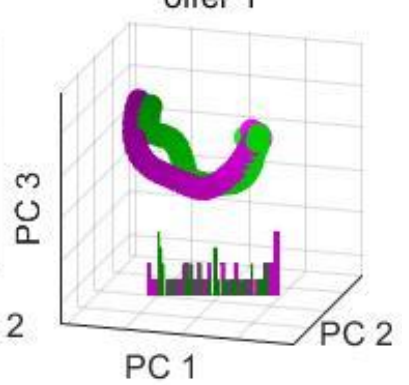

low reward rate high reward rate

B

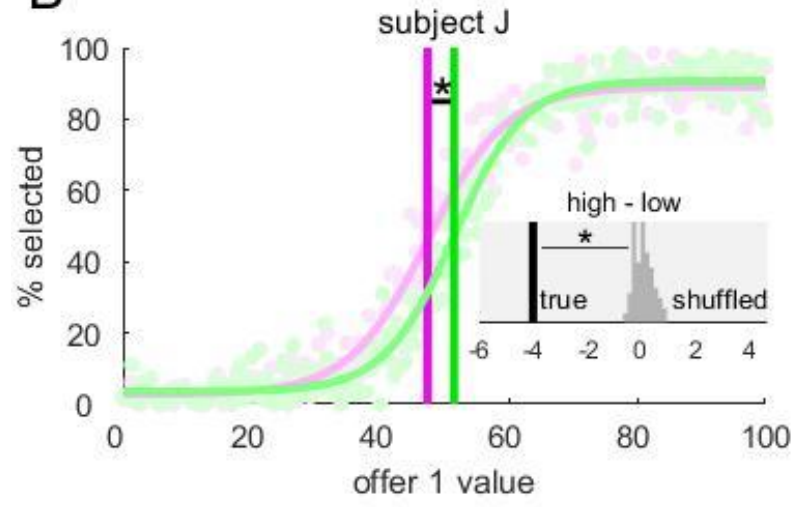

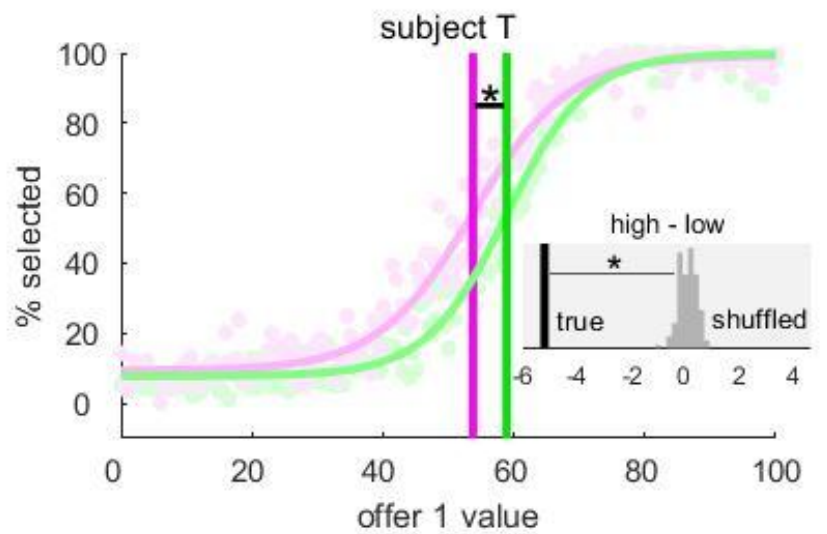

Figure 2. Discriminability of reward rate is higher at ITI than task-on period, and subjects behaviorally exploit reward rate information. (A) Upper panels:

Discriminability of high versus low reward rate in top 3 PCs over time. Discriminability (d') at the midpoint of the ITI epoch is significantly higher than d' at the midpoint of the trial epoch in both subjects. Error bars represent standard deviation. Lower panels: Projection of principal components for high (green) and low (magenta) reward rates into a 3D space, where each point represents the top three principal components resulting from a PCA conducted on a 500 ms period centered around one time point. There are 50 time points for each condition, describing the 500 ms epoch centered on the midpoint of the ITI and trial epochs. Histograms depict the distances of each point from the plane that separates the two groups of points, as determined by linear discriminant 
analysis. (B) Subjects' behavioral threshold for accepting an offer changes as recent reward rate changes: behavior threshold is higher for high reward rates than low reward rates for both subjects. Insets: the difference between the high reward rate threshold and low reward rate thresholds is significantly greater than the difference resulting from shuffled data.

\section{Dimensionality of neural subspace is greatest during the inter-trial-interval}

We next examined the relationship between ITI and trial epoch ensemble dimensionality.

217 Ensembles of neurons have constrained firing rate patterns; that is, single neurons have a limited

218 range of firing rates they can take and this range is determined in part by the responses of other

219 neurons. That range, collectively, defines the neural manifold or subspace of the population

220 (Gallego et al., 2017; Fusi et al., 2016). Dimensionality, then, refers to the number of the axes

221 within the larger space spanned by the subspace. It is a useful measure because it provides a

222 potential proxy for the complexity of processing going on within an area. Among other things,

223 greater dimensionality also allows for more ready separation of response patterns, thus allowing

224 for more abstract processing and learning (Fusi et al., 2016).

There are many ways to quantify the dimensionality of a subspace. We used a previously

226 developed method (Machens et al., 2010; Rouse \& Schieber, 2018). Specifically, we defined

227 dimensionality as the number of principal components it takes to explain $90 \%$ of the variance in

228 the neural activity at any particular moment (Figure 3A). We found that dimensionality is

229 substantially greater during the ITI epoch than during the trial epoch in both subjects (subject J:

23010.43 dimensions to 5.11 dimensions; $\mathrm{p}<0.001$, paired-t-test; subject T: 10.90 dimensions to

2315.32 dimensions, $\mathrm{p}<0.001$, paired t-test). (Figure 3B, upper panels). To compute these values

232 and test significance, we conducted a bootstrap in which we left out $25 \%$ of our sample each 
233 time we computed this dimensionality value (using the remaining 75\%) and averaged these

234 values (Figure 3B, lower panels).

235 To gain deeper insight into these processes, we next asked whether this change in

236 dimensionality is limited to the ITI vs. task periods. We hypothesized that if it reflects variations

237 in focus, we should be able to see changes as focus changes within the task itself (cf. Hayden et

238 al., 2009). For example, post-reward periods should have greater task focus than search periods

239 because they involve greater local focus (on consumption), while search periods involve

240 relatively more global focus (because they involve a search-like contemplation of multiple

241 options). Our data are consistent with this hypothesis. We compared the dimensionality of the

242 trial epoch between accepted and rejected offers using the same method specified in Figure 3B.

243 Viewing an accepted offer (which led to a reward consumption period) resulted in a significantly

244 lower dimensionality than viewing a rejected offer (which continued the trial); the effect was

245 observed in both subjects (Figure 3C, subject J: accepted offers 4.31 dimensions, rejected offers

2465.54 dimensions, $\mathrm{p}<0.001$, unpaired T-test; subject T: accepted offers 4.67 dimensions,

247 rejected offers 8.54 dimensions, $\mathrm{p}<0.001$, unpaired T-test). 


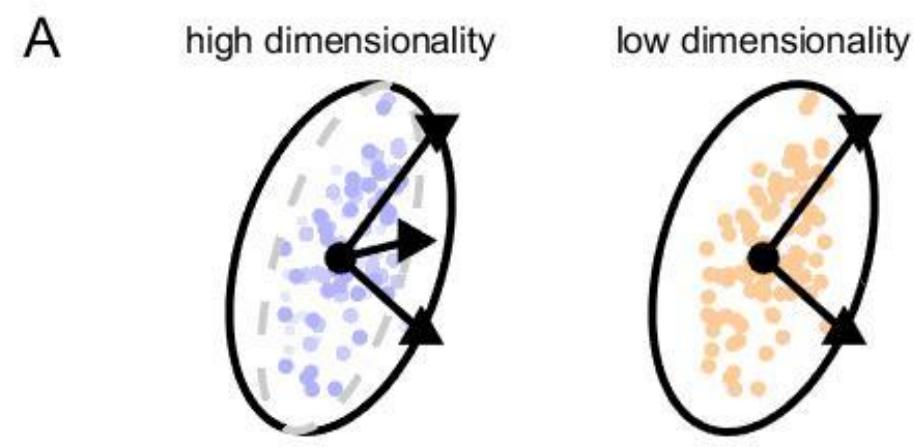

B

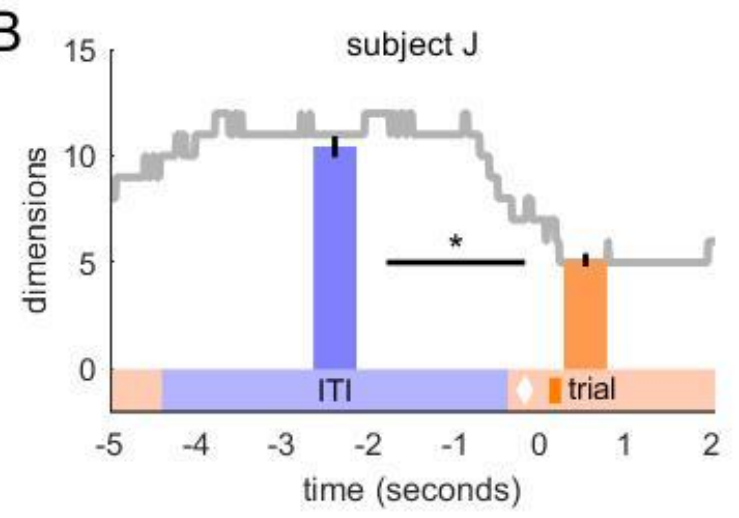

C

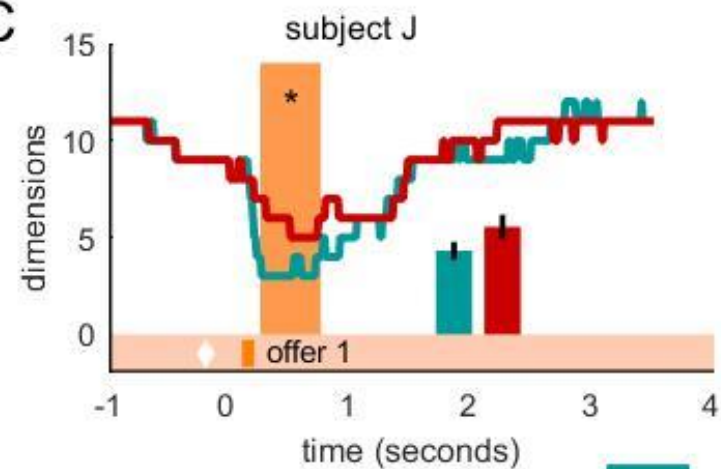

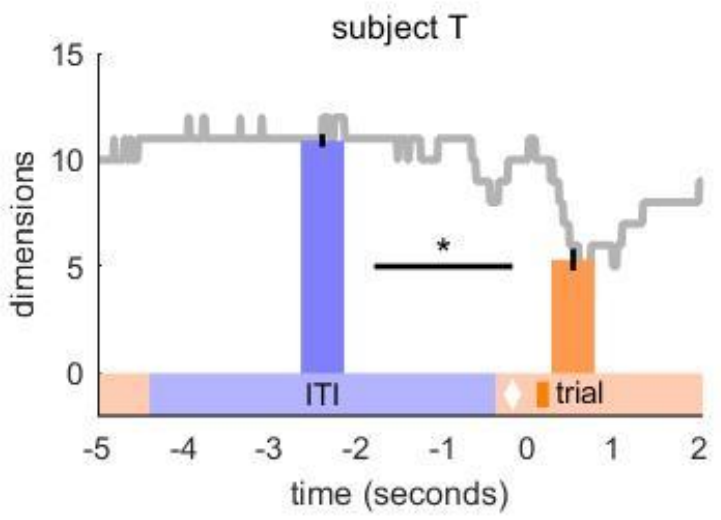

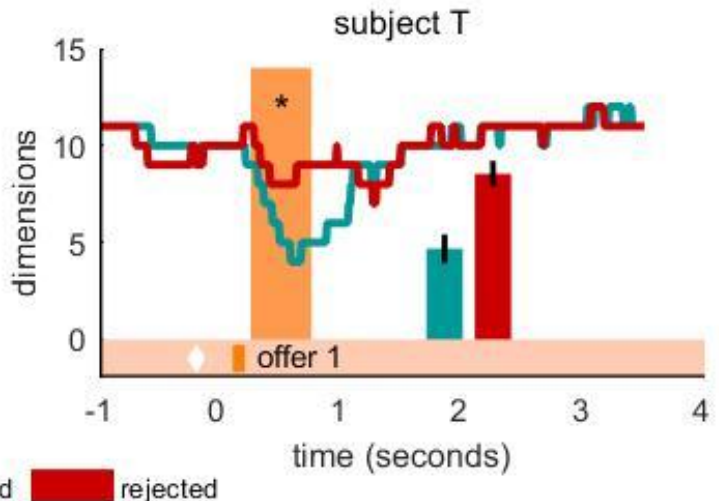

Figure 3. Subspace dimensionality is higher in the ITI than during the trial. (A) Schematic representing high and low dimensionality subspaces. (B) Dimensionality is significantly higher during the ITI epoch than the trial epoch for both subjects. Each point on gray line represents dimensionality of subspace over the preceding $500 \mathrm{~ms}$. Blue and orange bars represent the dimensionality values centered on the ITI epoch and trial epoch generated by bootstrapped data. Error bars are standard deviation. (C) Dimensionality is significantly lower for accepted offers (teal) than rejected offers (red) for both subjects. Teal and red lines represent dimensionality at each point in time as in (B). Bars represent dimensionality centered on the midpoint of the trial epoch for accepted and rejected offers, using bootstrapped data. Error bars are standard deviation. 


\section{Reduction in dimensionality is not explained by task-driven firing rate changes}

273 dimensionality is significantly higher than the true dimensionality (subject J: 7.57 dimensions

274 versus 5.35 dimensions, $\mathrm{p}<0.001$; subject T: 8.30 vs 7.33 dimensions, $\mathrm{p},=0.003$ ). This result

275 suggests that while some of the reduction in dimensionality could be due to the trial-driven firing

276 rate transient, a large part of it is independent of that.

\section{Reorganization rate of neural subspace reorganization is greatest during the inter-trial-}

\section{interval}

282 (Elsayed et al., 2016; Jiang et al., 2020; Tang et al., 2020; Yoo \& Hayden, 2020) Here, we refer 
(and its converse, low stability). Changes in neural activity patterns during rest states are generally considered to be slow $(<0.1 \mathrm{~Hz}$; Cabral et al., 2014; Deco \& Jirsa, 2012; de Pasquale \&

287 Marzetti, 2019). That is, techniques ranging from functional magnetic resonance imaging to magnetoencephalography, to electrophysiology, have revealed that low-frequency patterns characterize neural activity during rest. As a result, one would expect the pattern of changes we find during the ITI to be slow as well. However, there is emerging evidence of rapid computations during rest broadly across the default mode network (Baker et al., 2014; Khanna et al., 2015), and specifically in the motor cortex (Vidaurre et al., 2016). Here we provide evidence of fast-changing neural patterns (significant reorganization of the neural subspace between 500 ms epochs) during rest in the ventromedial prefrontal cortex. These results point to a changing view of default mode activity as not only slow oscillatory activity patterns, but also fastchanging dynamic activity.

To assess the subspace reorganization rate, we measured the similarity of the eigenvectors between each pair of subspaces before and after every given time point (10 ms intervals) of the task with $500 \mathrm{~ms}$ epochs (Methods). We considered every timepoint from the beginning of the ITI through two seconds after the reveal of the first offer. For each timepoint, we defined two time periods - $500 \mathrm{~ms}$ before, and $500 \mathrm{~ms}$ after - and computed the PCA coefficients for both time periods. We then projected the coefficients from the before time period into the after subspace and determined the percentage of after variance that was explained by the

304 before coefficients (Elsayed et al., 2016; Yoo \& Hayden, 2020; Jiang et al., 2020; Khanna et al., 2019). This overlap, expressed as a percentage, indicates the amount of reorganization of the neural subspace. A reorganization rate of $100 \%$ means the before subspace coefficients explained none of the after subspace (fast reorganization), and thus has completely reorganized, 
308 while $0 \%$ velocity means the before subspace explains all of the after subspace, and thus has not 309 reorganized at all.

310 We found that the subspace reorganization rate is significantly greater during the ITI than

311 during the task for both subjects (Figure 4B). Specifically, in subject $\mathrm{J}$ the reorganization for

312 sequential epochs at the midpoint of the ITI was $66.59 \%$ but only $28.34 \%$ at the midpoint of the

313 trial epoch $(\mathrm{p}<0.001$, one-sample t-test between true difference and shuffled differences, see

314 Methods). For subject T, these numbers were $72.76 \%$ for the ITI epoch and $55.35 \%$ for the trial

315 epoch $(\mathrm{p}<0.001$, one-sample t-test between true difference and shuffled differences). In other

316 words, vmPFC neuronal subspace occupancy changes much more over time during the ITI than

317 during the task; once the trial begins, however, the neural subspace becomes much more stable.

318 Finally, we note that the ITI subspace is organized drastically differently from the trial

319 subspace. That is, we conducted the same projection analysis on the $500 \mathrm{~ms}$ epoch centered

320 around the midpoint of the ITI and the $500 \mathrm{~ms}$ epoch centered around the midpoint of the trial

321 epoch, and found that the PC space of the ITI epoch changes $83.07 \%$ in subject $\mathrm{J}$ and $76.76 \%$ in

322 subject $\mathrm{T}$. 
A

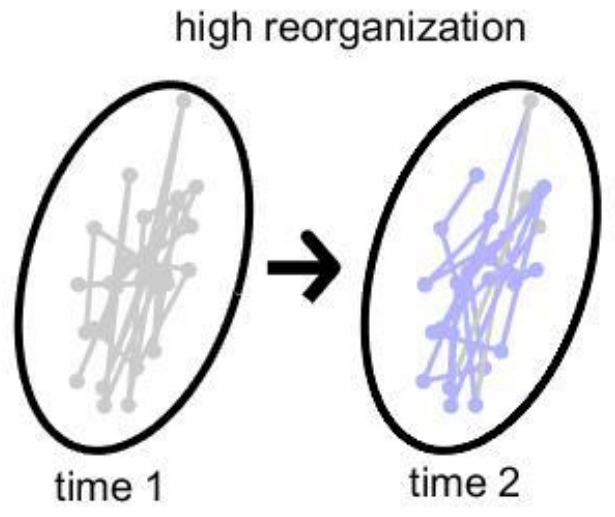

B
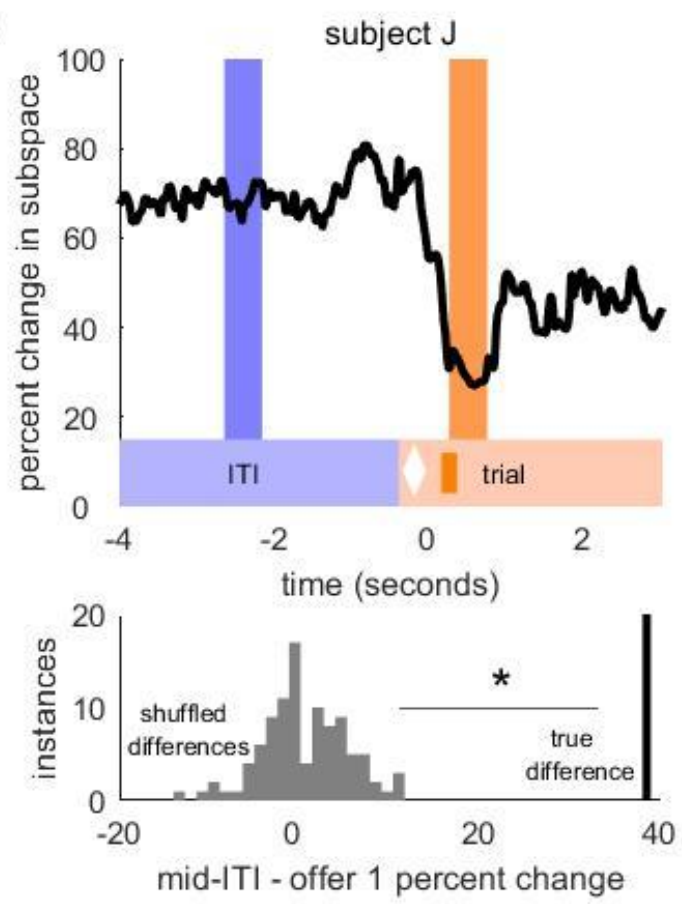

low reorganization

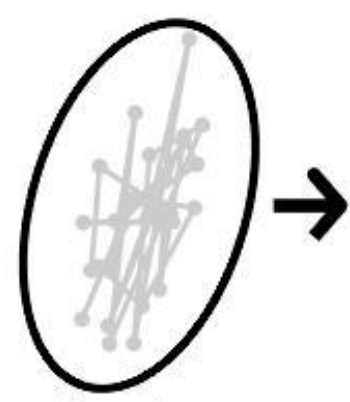

time 1

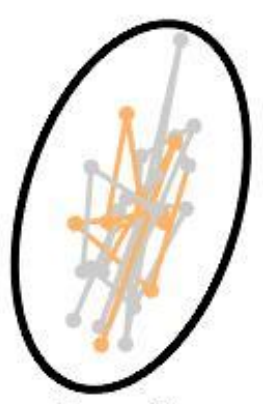

time 2

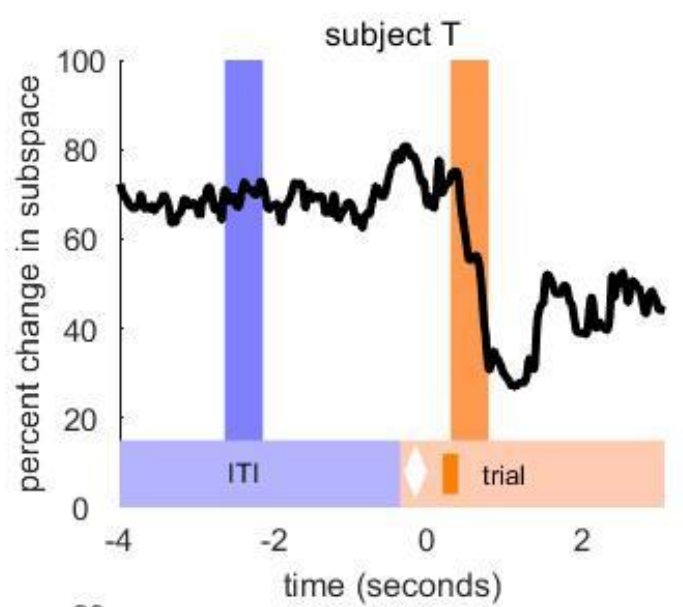

323

324

325

326

327

328

329

330

331

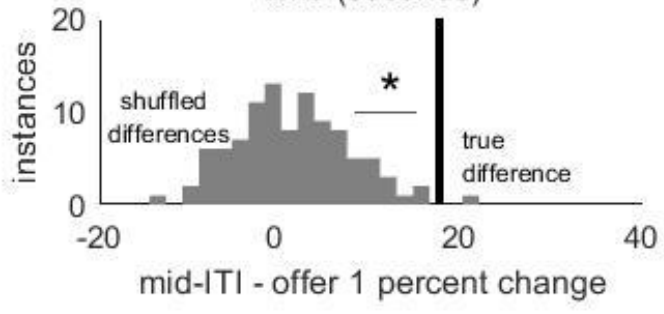

Figure 4. Subspace reorganizes much more during ITI than during trial. (A) Schematic of high subspace reorganization versus low subspace reorganization. (B) Upper panels: The percent change in subspace over the midpoint of the ITI epoch is greater than that over the midpoint of the trial epoch in both subjects. Lower panels: this difference is significantly greater than the distribution of differences generated by shuffling data 100 times. 


\section{DISCUSSION}

The ITI, and globally focused cognition more generally, occupies a large amount of our

335 lives and yet has not been studied nearly as much as task-oriented locally focused cognition.

336 Indeed, despite its quotidian nature, it remains strangely inaccessible to many conventional

337 cognitive neuroscientific methods (Raichle, 2006). Nonetheless, studies of default mode

338 processing have amply demonstrated that the time between trials and the times of global

339 cognition more generally are not simply "idling" or "paused" time in the brain (Boly et al., 2007;

340 Konishi et al., 2015; Weissman et al., 2006; Smallwood et al., 2013; , Baird et al., 2013 ).

341 Instead, the brain makes use of this time to engage in a variety of cognitive functions. Many of

342 these seem to be related to mnemonic processing.

343 Here, we examined the responses of a population of neurons in area 14 of the vmPFC

344 while macaques performed a foraging search task (Mehta et al., 2019). We imposed an unusually

345 long interval between sequential trials (duration of 4 seconds), a design feature that allowed us to

346 compare neuronal ensemble responses during task-on (trial) and task-off (inter-trial interval, ITI)

347 periods while avoiding immediate post-trial and pre-trial effects. We examined encoding of a key

348 piece of mnemonic information in the task - recent reward rate, which we show are attended and

349 learned. During the ITI, that information is encoded in a more separable format: an effect we

350 demonstrate with our discriminability analysis. In other words, information is present during

351 both the task and ITI epochs, but it is more untangled during the ITI (DiCarlo et al., 2012; Yoo \&

352 Hayden, 2018; DiCarlo \& Cox, 2007). This finding suggests that the brain actively reformats

353 task-relevant information depending on task context and, in particular, that it makes mnemonic

354 information more functionally available during the ITI than during the task itself. 
Why would reward rate information be more separable during the ITI than during the task itself? During the trial, the subject must do two things - (1) apply learned information for

357 purposes of guiding decisions and (2) be alert for new incoming information. Both processes

358 may be antagonistic to the kinds of neural processes that are associated with mnemonic

359 processing, including computing and representing the recent average reward value. In other

360 words, explicit reward value representation is a guide for behavior but it is conceptually quite

361 distinct from its implementation, and cognition may have a finite capacity. It may be efficient,

362 then, to process it asynchronously during low-demand periods. Indeed, we hypothesize that the

363 more accessible format during the delay reflects the need to process it and develop a decision

364 strategy; that strategy only needs to be implemented during choice, so the information that

365 guides the strategy can be stored in a more latent manner.

We also found that the ITI is associated with more complex processing, as indicated by

367 two complementary measures: subspace dimensionality and subspace change rate.

368 Dimensionality refers to the volume occupied by an ensemble in a high-dimensional space: a

369 higher dimension is presumed to reflect less redundancy and more complex and multifarious

370 response patterns (Cunningham \& Byron, 2014). Subspace change rate, on the other hand is a

371 new measure that we introduce here, but it is one that follows naturally from past work on

372 subspace reorganization - it is, essentially the rate at which reorganization occurs. We reasoned

373 that faster reorganization may imply rapid cognitive processing with multiple changing priorities.

374 Some of the dimensionality and velocity results are likely related to the specific mnemonic

375 function discussed above, but some likely reflect even more complex processing to which we

376 don't have experimental access. 
Why would the brain alternate between processing different types of information in

378 different modes? We speculate that the brain is not a strictly parallel processor, and that its

379 capacity is highly restricted (Shenav et al., 2017). Specifically, we conjecture that broad and

380 narrow-focused attention naturally competes and cannot be effectively implemented at the same

381 time. In other words, we propose that information processing is analogous to preemptive

382 multiple processing in computer science. This type of multiple processing operates by

383 prioritizing the task and context switch operation allows the interruption of certain tasks and

384 resumes later. The global information processing can be interrupted by task engagement (local

385 phase) and resumed once the individual occasion of search ends. According to preemptive

386 multiple processing, the operating processor never shuts down but they switch the information

387 being processed. 
We conducted all procedures in compliance with the Public Health Service's Guide for the Care and Use of Animals and approved by the University Committee on Animal Resources at the University of Rochester. These data were previously analyzed in a published study (Mehta et al., 2019). Subjects were two male rhesus macaques (Macaca mulatta: subject J age 10 years; subject $\mathrm{T}$ age 5 years). To maintain head position, we used a small titanium prosthesis. We trained the subjects first to habituate to laboratory conditions and then to perform oculomotor tasks for liquid reward. To record neural data, we placed a Cilux recording chamber (Crist instruments) over the ventromedial prefrontal cortex (vmPFC) of each animal. We verified the accuracy of this position using magnetic resonance and a Brainsight system (Rogue Research Inc.). We provided the animals with all appropriate analgesics and antibiotics post-procedure, and regularly sterilely cleaned and sealed the implanted chamber.

\section{Recording site}

We performed all recordings between morning and midday (10am to 3pm), using a standard recording grid (Crist Instruments) to approach area 14 (vmPFC) as described by the Paxinos atlas (Paxinos et al., 2000). Specifically, we recorded from neurons located between 42 and $31 \mathrm{~mm}$ rostral to the interaural plane, 0 and $7 \mathrm{~mm}$ horizontally from the brain's ventral surface, and 0 to $7 \mathrm{~mm}$ sagittal from the medial wall.

\section{Electrophysiological techniques, eye tracking and reward delivery}

All methods used have been described previously and are summarized here (Strait et al., 2014). We used a microdrive (NAN instruments) to guide single electrodes (Frederick Haer \& Co., impedance range 0.7 to $5.5 \mathrm{MU}$ ). Each recording session, we isolated between one and four neurons on a Plexon system (Plexon, Inc.). We used data from all neurons we could isolate for at least 300 consecutive trials, regardless of their behavior during the task. In practice, $86 \%$ of neurons had over 500 trials

To obtain eye position data at $1,000 \mathrm{~Hz}$, we used an infrared eye-monitoring camera system (SR Research). We controlled the task presentation with a computer running Matlab (Mathworks) with Psychtoolbox and Eyelink Toolbox. Our visual stimuli were colored shapes on a computer monitor located $60 \mathrm{~cm}$ from the animal at eye level. We controlled liquid reward delivery with a standard solenoid valve.

\section{Experimental Design}

Subjects performed a diet-search task (Figure 1) that is described in detail in a previous publication (Mehta et al., 2019). The task is a conceptual extension of previous foraging tasks we have developed. Each trial, subjects were presented with either four or seven white diamonds that appeared in random positions on the screen. $400 \mathrm{~ms}$ of continuous fixation on one of these diamonds caused it to disappear and reveal a reward offer underneath. Reward offers were partially-filled-in orange bars that indicated riskless reward amount by the proportion of their area that was filled in. There were 200 possible reward offer sizes per offer. To accept an offer, subjects had to continuously fixate on one for $300 \mathrm{~ms}$.

Subjects freely searched through the diamonds in any order, but had to accept an offer to obtain a reward and end the trial. Rejection of an offer (breaking fixation before $300 \mathrm{~ms}$ had elapsed) resulted in a continuation of the trial (free search through the diamonds). Reward was 
delivered immediately after acceptance of an offer, which was followed by a four-second intertrial-interval.

Previous training history for these subjects included two types of foraging tasks (Blanchard and Hayden, 2015; Blanchard et al., 2015), complex choice tasks with time elements (Blanchard et al., 2014), two types of gambling tasks (Farashahi et al., 2018; Azab and Hayden, 2017), attentional tasks (similar to those in Hayden and Gallant, 2013), and two types of rewardbased decision tasks (Pirrone et al., 2018; Wang and Hayden, 2017).

\section{Statistical Analysis for Physiology}

We formed Peri-Stimulus Time Histograms (PSTHs) by aligning spike rasters to each offer reveal of each trial and averaging firing rates across multiple trials. Firing rates were normalized (where indicated) by subtracting the mean and dividing by the standard deviation of the entire neuron's PSTH.

\section{Analysis epochs}

Before beginning analysis, we determined two epochs over the course of the trial to correspond to the ITI and the task. We selected the $500 \mathrm{~ms}$ period in the middle of the 4-second ITI to represent the ITI epoch, and the $500 \mathrm{~ms}$ in the middle of the 1-second epoch following the reveal of the first offer to represent the offer epoch.

\section{Creating the PC spaces}

To create a PC-space for the ITI and first offer of each trial, we began with the PSTHs of all the trials, sorted into two groups: trials where the subject accepted the first offer, and trials where the subject rejected the first offer. These PSTHs were Gaussian smoothed over 15 time bins $(150 \mathrm{~ms})$, and normalized. (Note that while data was divided into accept and reject data for the dimensionality and orthogonality analyses, it was divided into low and high reward rate datasets for the discriminability analysis).

We then found the mean PSTH for each cell for each of these groups, giving us two matrices per subject - each with cells as rows and time points of the PSTH as columns. In other words, we computed the average behavior of each cell for trials where the subject accepted the first offer, and trials where the subject rejected the first offer. We then appended the reject matrix to the accept matrix to create a combined matrix.

To obtain PC spaces over the course of the ITI and first offer, we used a sliding boxcar of 50 time bins $(500 \mathrm{~ms})$ to perform a PCA on each $500-\mathrm{ms}$ epoch of PSTH data from the beginning of the ITI through 2 seconds following the reveal of the first offer.

To obtain PC spaces for trials following the acceptance or rejection of an offer, we used a sliding boxcar of 50 time bins to perform a PCA on each 500-ms epoch of PSTH data from 2 seconds before the reveal of each offer to 3 seconds after.

\section{Discriminability analysis}

We defined discriminability between two task conditions as the separation between representations of those variables in principal component space. To this end, we performed PCAs as described above, using neural data with each trial split into either a low-reward rate (between 0 and $71 \%$ for subject J; 0 and $72 \%$ for subject T, see Mehta 2019 for calculation of reward rate), or high-reward-rate ( $71 \%-100 \%$ for subject $\mathrm{J} ; 72 \%$ and above for subject T) condition. We then obtained the top three principal components (the principal components that 
explain the three largest amounts of variance in the neural activity) for each condition and used a linear discriminant analysis (LDA) to assess their separability.

Specifically, we plotted the top three principal components for each condition over a specific time epoch, so that we obtained a cloud of three-dimensional points representing all the time points in one epoch for each condition. Then, the LDA provided us with a plane separating the two clouds of points. To obtain $d^{\prime}$, the separability between these two clouds, we first calculated the distance of each of these points from the separating plane, as well as the standard deviations of the distributions of distances for each condition. We defined $d$ ' as follows:

$$
d^{\prime}=\mu_{1}-\mu_{2} / \sqrt{ }\left(\sigma_{1}{ }^{2}-\sigma_{2}{ }^{2}\right)
$$

Where $\mu_{1}$ and $\mu_{2}$ are the means of the distances for each condition (low and high reward rate) and $\sigma_{1}$ are $\sigma_{2}$ are the standard deviations of these distributions.

\section{Dimensionality analysis}

We defined dimensionality of an epoch as the number of principal components it takes to explain $90 \%$ of the variance in the neural activity during that epoch (Elsayed et al., 2016; Yoo \&Hayden, 2020). We used the PCAs run as detailed above on our epochs of interest and obtained the percentage of the variance that each PC explained, sorted from greatest variance explained to least. For each epoch of interest, we found the number of principal components, starting with the one that explained the most variance, that it took to explain a minimum of $90 \%$ of the total variance. This value we termed the 'dimensionality.'

It is important to note that dimensionality can only be a whole number: it represents a number of principal components. When we report dimensionality as having decimal values, it is because we have averaged several whole numbers over a period of time. We interpret these decimal values as estimates of dimensionality.

To perform significance tests on dimensionality data, we had to bear in mind that each sequential time point's dimensionality is created from data that overlaps with the next time point. Thus, we could not perform a simple t-test between, for example, the dimensionalities of the time points between the reveal of the first offer and one second following. Instead, we used a bootstrap: we selected the central time point of each analysis epoch (which represents data from $250 \mathrm{~ms}$ before that point and $250 \mathrm{~ms}$ after) and recalculated it 100 times using $75 \%$ of the original data each time. We then performed a t-test between these two groups of 100 points between epochs.

To create control data, we randomly swapped the PSTHs of the offer 1 epoch between cells. For example, if there were three real cells, Cell A, B and C and three control cells, Cell A', B', and C, Cell A' may have the offer 1 epoch of Cell C', Cell B' might have the offer 1 epoch of Cell A, and Cell $\mathrm{C}$ would have the offer 1 epoch of Cell B. Thus, the control data would have the same mean firing rate as the real data. However, suppose that the combination of Cells A and B specifically is meaningful to the vmPFC; that is, population information is meaningful. Now, the combination of Cell A and Cell B's firing rate is meaningless, because their PSTHs have been swapped out with those of other cells. Thus, our control data was such that the mean firing rate at every time point of the offer 1 epoch was the same, but the properties of the data at the population level are meaningless.

\section{Orthogonality analysis}


To estimate the amount of reorganization between subspaces for the two epochs, we did the following (Elsayed et al., 2016; Yoo \& Hayden, 2020). We first performed principal

527 components analysis (PCA) on the matrix P1 for each epoch (see above) to obtain the epoch-1

528 PCs. For the remainder of the analysis, we used only the top ten PCs resulting from each PCA.

529 We obtained the top ten epoch 2 PCs by performing PCA on matrix P2. To examine the

530 relationship between the subspaces, we projected the epoch 1 activity onto the epoch 2 PCs and

531 quantified the percent of variance explained relative to the total variance of epoch 1.

532 To perform significance tests on orthogonality data, we noted that orthogonality values

533 are derived from principal components that describe overlapping epochs of neural data. Thus

534 again, we could not perform a simple t-test between the average orthogonality of different

535 epochs. Instead, we created shuffled data by scrambling the PCA inputs between the two epochs

536 of interest, 100 times. We then calculated the orthogonality of the central time point of the ITI

537 and the offer epoch 100 times with these sets of scramble data. We compared the true difference

538 in orthogonality to the distribution of shuffled differences with a t-test to obtain a p-value. 


\section{REFERENCES}

542 Azab, H., \& Hayden, B. Y. (2017). Correlates of decisional dynamics in the dorsal anterior

543 cingulate cortex. PLoS biology, 15(11), e2003091. networks in anterior prefrontal cortex support metacognitive ability for memory and perception. Journal of Neuroscience, 33(42), 16657-16665.

Baker, A. P., Brookes, M. J., Rezek, I. A., Smith, S. M., Behrens, T., Smith, P. J. P., \& Woolrich, M. (2014). Fast transient networks in spontaneous human brain activity. Elife, 3, e01867. Neuron, 100(2), 490-509. anterior cingulate neurons during persistent commitment to a decision. Journal of neurophysiology, 114(4), 2439-2449.

Blanchard, T. C., \& Hayden, B. Y. (2015). Monkeys are more patient in a foraging task than in a standard intertemporal choice task. PloS one, 10(2), e0117057. (2007). Baseline brain activity fluctuations predict somatosensory perception in humans. Proceedings of the National Academy of Sciences, 104(29), 12187-12192. brain activity during rest. Progress in neurobiology, 114, 102-131. behavioral performance on individual trials. Journal of Neuroscience, 30(45), 15241-15253. 
Cunningham, J. P., \& Byron, M. Y. (2014). Dimensionality reduction for large-scale neural recordings. Nature neuroscience, 17(11), 1500-1509.

Daselaar, S. M., Prince, S. E., \& Cabeza, R. (2004). When less means more: deactivations during encoding that predict subsequent memory. Neuroimage, 23(3), 921-927.

Deco, G., \& Jirsa, V. K. (2012). Ongoing cortical activity at rest: criticality, multistability, and ghost attractors. Journal of Neuroscience, 32(10), 3366-3375.

de Pasquale, F., \& Marzetti, L. (2019). Temporal and spectral signatures of the default mode network. Magnetoencephalography: From Signals to Dynamic Cortical Networks, 571-603.

DiCarlo, J. J., \& Cox, D. D. (2007). Untangling invariant object recognition. Trends in cognitive sciences, 11(8), 333-341.

DiCarlo, J. J., Zoccolan, D., \& Rust, N. C. (2012). How does the brain solve visual object recognition?. Neuron, 73(3), 415-434.

Elsayed, G. F., Lara, A. H., Kaufman, M. T., Churchland, M. M., \& Cunningham, J. P. (2016). Reorganization between preparatory and movement population responses in motor cortex. Nature communications, 7(1), 1-15.

Farashahi, S., Azab, H., Hayden, B., \& Soltani, A. (2018). On the flexibility of basic risk attitudes in monkeys. Journal of Neuroscience, 38(18), 4383-4398.

Ferguson, T. S. (1989). Who solved the secretary problem?. Statistical science, 4(3), 282-289.

Fusi, S., Miller, E. K., \& Rigotti, M. (2016). Why neurons mix: high dimensionality for higher cognition. Current opinion in neurobiology, 37, 66-74.

Gallego, J. A., Perich, M. G., Miller, L. E., \& Solla, S. A. (2017). Neural manifolds for the control of movement. Neuron, 94(5), 978-984.

Hayden, B. Y., Nair, A. C., McCoy, A. N., \& Platt, M. L. (2008). Posterior cingulate cortex mediates outcome-contingent allocation of behavior. Neuron, 60(1), 19-25.

Hayden, B. Y., Pearson, J. M., \& Platt, M. L. (2009). Fictive reward signals in the anterior cingulate cortex. science, 324(5929), 948-950. 
Hayden, B. Y., Smith, D. V., \& Platt, M. L. (2009). Electrophysiological correlates of defaultmode processing in macaque posterior cingulate cortex. Proceedings of the National Academy of Sciences, 106(14), 5948-5953. decisions in a patchy environment. Nature neuroscience, 14(7), 933.

Hayden, B., \& Gallant, J. (2013). Working memory and decision processes in visual area v4. Frontiers in neuroscience, 7, 18.

Hayden, B. Y., \& Platt, M. L. (2010). Neurons in anterior cingulate cortex multiplex information about reward and action. Journal of Neuroscience, 30(9), 3339-3346. Annual review of neuroscience, 39, 149-170. nonhuman primates. Psychonomic bulletin \& review, 23(2), 593-600. Single Neurons. Cell reports, 32(6), 108006. (2014). Damage to the salience network and interactions with the default mode network. Journal 647 of neuroscience, 34(33), 10798-10807.

649 Kao, T. C., Sadabadi, M. S., \& Hennequin, G. (2020). Anticipatory control of movement in a 650 thalamo-cortical circuit model. bioRxiv.

652 Keemink, S. W., \& Machens, C. K. (2019). Decoding and encoding (de) mixed population 
Khanna, S. B., Snyder, A. C., \& Smith, M. A. (2019). Distinct sources of variability affect eye movement preparation. Journal of Neuroscience, 39(23), 4511-4526. by the past: the default mode network supports cognition that is independent of immediate perceptual input." PloS one 10, no. 6 (2015): e0132209. "what" and "when" in the prefrontal cortex. Journal of Neuroscience, 30(1), 350-360. (2007). Wandering minds: the default network and stimulus-independent thought. Science, 315(5810), 393-395. (2001). Cortical networks for working memory and executive functions sustain the conscious resting state in man. Brain research bulletin, 54(3), 287-298.

675

678 Neuroscience, 39(27), 5336-5350.

Northoff, G., Qin, P., \& Nakao, T. (2010). Rest-stimulus interaction in the brain: a review. coordinates. speed-value trade-off: Human and monkey decision making is magnitude sensitive. Decision, $6885(2), 129$. 314(5803), 1249. 190.

697 433-447. 

shift during reach to grasp. Cell reports, 25(11), 3158-3168.

Smallwood, J., Tipper, C., Brown, K., Baird, B., Engen, H., Michaels, J. R., ... \& Schooler, J. W. (2013). Escaping the here and now: evidence for a role of the default mode network in perceptually decoupled thought. Neuroimage, 69, 120-125.

Stephens, D. W., \& Krebs, J. R. (1986). Foraging theory (Vol. 1). Princeton University Press. inhibition in ventromedial prefrontal cortex. Neuron, 82(6), 1357-1366. representation of value in the parietal cortex. science, 304(5678), 1782-1787. dependent activity subspaces for working memory and motor preparation in the lateral prefrontal cortex. eLife, 9, e58154.

Wang, M. Z., \& Hayden, B. Y. (2017). Reactivation of associative structure specific outcome $1-13$. of momentary lapses in attention. Nature neuroscience, 9(7), 971-978. actions. Neuron, 99(3), 434-447. neural subspace reorganization in core reward regions. Neuron, 105(4), 712-724. 
bioRxiv preprint doi: https://doi.org/10.1101/2020.10.08.331579; this version posted October 8, 2020. The copyright holder for this preprint (which was not certified by peer review) is the author/funder. All rights reserved. No reuse allowed without permission.

738 Zou, Q., Ross, T. J., Gu, H., Geng, X., Zuo, X. N., Hong, L. E., ... \& Yang, Y. (2013). Intrinsic

739 resting state activity predicts working memory brain activation and behavioral performance.

$740 \quad$ Human brain mapping, 34(12), 3204-3215. 